\title{
On the origin of noisy states whose teleportation fidelity can be enhanced through dissipation
}

\author{
Somshubhro Bandyopadhyay円 \\ Department of Physics, Bose Institute, 93/1 A. P. C Road, Calcutta - 700009, India
}

\begin{abstract}
Recently Badziag et al. [1] obtained a class of noisy states whose teleportation fidelity can be enhanced by subjecting one of the qubits to dissipative interaction with the environment via amplitude damping channel (ADC). We show that such noisy states result while sharing the states $\left|\Phi^{ \pm}\right\rangle=\frac{1}{\sqrt{2}}(|00\rangle \pm|11\rangle)$ across ADC. We also show that under similar dissipative interactions different Bell states give rise to noisy entangled states that are qualitatively very different from each other in the sense, only the noisy entangled states constructed from the Bell states $\left|\Phi^{ \pm}\right\rangle$can be made better sometimes by subjecting the unaffected qubit to a dissipative interaction with the environment. Importantly if the noisy state is non teleporting then it can always be made teleporting with this prescription. We derive the most general restrictions on improvement of such noisy states assuming that the damping parameters being different for both the qubits. However this curious prescription does not work for the noisy entangled states generated from $\left|\Psi^{ \pm}\right\rangle=\frac{1}{\sqrt{2}}(|01\rangle \pm|10\rangle)$. This shows that an apriori knowledge of the noisy channel might be helpful to decide which Bell state needs to be shared between Alice and Bob.
\end{abstract}

\section{Introduction}

Quantum entanglement [2], is a property of bipartite systems by virtue of which the spatially separated subsystems exhibit strong correlations among themselves that cannot be explained in classical terms. Often termed as quantum nonlocality, the subject had been

\footnotetext{
1 som@ee.ucla.edu, dhom@boseinst.ernet.in
} 
under extensive study and debate since 1935 when Einstein, Podolsky and Rosen questioned the completeness of quantum mechanics [3]. Later, in 1964 in his pioneering work John Bell [5] showed that such nonlocal correlations are inherently quantum mechanical and any model admitting the description of local hidden variables (or one may prefer to say local realism) fails to explain such correlations. A consequence of quantum superposition principle, thus entanglement has been the most celebrated manifestation of quantum nonlocality [4].

In recent years there is a renewed interest in entanglement primarily because of its newly found applications in quantum information processing [6] and quantum computing [7] and as it turned out, many of the applications would have either been impossible or less efficient classically. A necessary condition for faithful implementation of a quantum information protocol is that the parties share a maximally entangled state (MES), each of them having access to their respective subsystem whereby they perform local quantum operations and communicate among themselves one way or both ways via classical channel to implement the concerned protocol. The local operations include unitary operations, von neuman measurements and generalized measurements which may involve ancillary systems Alice and Bob might prepare in their laboratories. For two qubit systems a class of maximally entangled states are called Bell states that are defined by

$$
\begin{aligned}
& \left|\Phi^{ \pm}\right\rangle=\frac{1}{\sqrt{2}}(|00\rangle \pm|11\rangle) \\
& \left|\Psi^{ \pm}\right\rangle=\frac{1}{\sqrt{2}}(|01\rangle \pm|10\rangle)
\end{aligned}
$$

The four Bell states are all equally good for faithful quantum communication. For instance, quantum teleportation [8] with fidelity one is achieved only with maximally entangled states, viz. by any one of the four Bell states. They also share the property of being local unitary equivalent (they are mapped onto each other by local pauli rotations). 
As noted above maximally entangled states are crucial for faithful information processing. In practice, however, entanglement is susceptible to local interactions with the environment. Such dissipative interactions may take place during encoding/decoding processes, transmission and sharing of entanglement. This give rise to a mixed entangled state or a separable state depending on the nature, strength and duration of the interaction. The noisy states thus formed are of little or no use for information processing. However one can still generate a fewer number of maximally entangled states from an ensemble of mixed entangled states by applying the distillation protocols [9].

Despite being responsible for destroying quantum coherence there is a genuine positive side of dissipative effects as shown recently by Badziag et al. [1]. They demonstrated that a family of non teleporting mixed entangled states can be made teleporting through dissipative intaeractions with the environment via amplitude damping channel. Here it may be worth mentioning that ADC is fairly rich in structure and was shown to be having many interesting properties. For instance Bennett et al. showed that if the noisy channel is ADC then by entangling the transmission bits one can increase the receiver's capability of correct inference [10].

In the present work we uncover some curious features when a pure but maximal entanglement interacts with the environment via ADC. The problem that we consider in this paper is the following. Suppose we have a bipartite maximally entagled state, say, $\rho$ ( $\rho$ being one of the four Bell states), and the qubits of the entangled pair undergo local interactions with their respective environment via amplitude damping channel. There are two possibilities:

Case 1: Only one qubit gets affected: $\rho \rightarrow \rho_{1}$ (a mixed entangled state).

Case 2: Both the qubits are affected: $\rho \rightarrow \rho_{2}$ (a mixed entangled state).

Typically, both the cases may arise while sending entanglement across a noisy channelthe so called transmission errors or due to some coding / decoding process via some noisy 
channel. Whatever be the source of errors we ask which of the two states $\rho_{1}$ and $\rho_{2}$, is more useful for teleportation?

Since both qubits are affected in the second case, one may be tempted to speculate that $\rho_{2}$ is always qualitatively worse that $\rho_{1}$ in terms of efficiency to perform teleportation. To our surprise we find that the state $\rho_{2}$ is sometimes "better" than $\rho_{1}$ having a higher teleportaion fidelity ! Quite interestingly this effect is observed only if the Bell state that we wish to share is either of the two states $\left|\Phi^{ \pm}\right\rangle$. The effect is also shown to depend on the strength of the amplitude damping channel. Moreover we show that if $\rho_{1}$ is a non teleporting state then it can always be made teleporting by subjecting the other qubit (which didn't interact with the environment before) to similar dissipative interaction. An interesting feature of this effect is that the teleportation fidelity of the noisy states that are formed while sharing the other two Bell states $\left|\Psi^{ \pm}\right\rangle$, cannot be enhanced by the same prescription. Here we note that since the process is trace preserving, we have thus been able to identify and parametrize the class of non teleporting states having fully entangled fraction $f$ [1] less than $1 / 2$, for which we can increase $f$ to greater than half so that they become suitable for distillation [9] without discarding any pair, as opposed to filtering procedures [12, 13]. This shows that an a priori knowledge of the noisy channel might be helpful to decide which Bell state needs to be shared between Alice and Bob.

As noted earlier, Badziag et al. [1] obtained a class of noisy states whose teleportation fidelity can be enhanced by subjecting one qubit to undergo dissipative interactions with its local environment via ADC. What is the origin of such noisy states? Our results show that the class of states obtained in Ref. [1] belong to the family of noisy states that result when any of the particular Bell states, namely $\left|\Phi^{ \pm}\right\rangle$interacts with the environment via the ADC.

This paper is arranged in the following way. In Sec. II we review some known results relating fully entangled fraction to teleportation fidelity and distillation. In Sec. III the 
action of an ADC on a qubit is reviewed. The generation of noisy states while sharing a Bell state across ADC is described in Sec. IV. In Sec. V we discuss the improvement of noisy states via dissipation. We conclude with some remarks and discussion in Sec. VI.

\section{Entanglement Fidelity, Fully Entangled Fraction, Teleportation Fidelity and Distillation threshold}

In this paper reliability for teleportation will be the criterion for judging the quality the noisy entangled states. The following results will be useful .

(1) Fully entangled fraction $f$ of a bipartite entangled state $\rho$ in $C^{2} \otimes C^{2}$ is defined as

$$
f(\rho)=\max _{\psi}\langle\psi|\rho| \psi\rangle
$$

where the maximum is taken over all maximally entangled states $|\psi\rangle$ [11]. For $\rho$ to be useful for quantum teleportation we must have $f>\frac{1}{2}$ [14, 15]. It was also shown that in the standard teleportation scheme [ 8 the maximal fidelity $F$ achievable is related to $f$ by [15]

$$
F=\frac{2 f+1}{3}
$$

(2) For the states with $f \leq \frac{1}{2}$ one cannot directly apply the distillation protocol by Bennett et al. [9]. For those states one first resorts to filtering procedures [12, 13] to enhance the value of $f$ from $f \leq \frac{1}{2}$ to $f>\frac{1}{2}$. 


\section{Action of the Amplitude Damping Channel on a Qubit}

Here we briefly review the action of ADC on a qubit. Details can be found in Ref. [16]. The amplitude damping channel describes the interaction of a two level atom with the electromagnetic field (environment). Specifically the decay of an excited state of a two level atom by spontaneous emission of a photon in presence of an e.m. field is what modelled by this channel. The unitary transformation that governs the evolution of the system and the environment (the environment can be always taken to be in some pure state without any loss of generality) is defined by,

$$
\begin{aligned}
|0\rangle_{A}|0\rangle_{E} & \rightarrow|0\rangle_{A}|0\rangle_{E} \\
|1\rangle_{A}|0\rangle_{E} & \rightarrow \sqrt{1-p}|1\rangle_{A}|0\rangle_{E}+\sqrt{p}|0\rangle_{A}|1\rangle_{E}
\end{aligned}
$$

Physically this implies that if an atom is in an excited state $|1\rangle_{A}$, with probability $p$ it makes a transition to the ground state $|0\rangle_{A}$ with the emission of a photon. The environment as a result also makes a transition from the "no-photon" state $|0\rangle_{E}$ to the "one-photon" state $|1\rangle_{E}$. Tracing out the environment we obtain the Kraus operators, with the Kraus operators $K_{i}:$

$$
K_{1}=\left(\begin{array}{cc}
1 & 0 \\
0 & \sqrt{1-p}
\end{array}\right), K_{2}=\left(\begin{array}{cc}
0 & \sqrt{p} \\
0 & 0
\end{array}\right)
$$

satisfying the completeness relation

$$
\sum_{i=1}^{2} K_{i}^{\dagger} K_{i}=1
$$


The density matrix $\rho$ of the quantum system then evolves as

$$
\rho \longrightarrow \rho^{\prime}=S(\rho)=\sum_{i=1}^{2} K_{i} \rho K_{i}^{\dagger}
$$

The above equation basically defines a linear map which takes a density matrix to another density matrix (a superoperator). It is clear that the amplitude damping channel is characterized by the parameter $p$, which denotes the dissipation strength when a qubit interacts with the environment via this channel.

\section{Sending Bell States across the Amplitude Damping Channel}

In this section we consider the problem of sending the Bell states across the amplitude damping channel. We assume that Alice prepares one of the Bell states locally and send one of the qubits to Bob. This qubit interacts with the environment via ADC during the transmission. The initial Bell state is thus transformed to a mixed entangled state. Let $\rho$

be the density operator representing one of the four Bell states, then the interaction with the environment via ADC is described by the following transformation:

$$
\rho \longrightarrow \widetilde{\rho}(p)=S(\rho)=W_{0} \rho W_{0}^{\dagger}+W_{1} \rho W_{1}^{\dagger}
$$

where $W_{i} \equiv I \otimes K_{i}$ are given by

$$
W_{0}=\left[\begin{array}{cccc}
1 & 0 & 0 & 0 \\
0 & \sqrt{1-p} & 0 & 0 \\
0 & 0 & 1 & 0 \\
0 & 0 & 0 & \sqrt{1-p}
\end{array}\right] ; W_{1}=\left[\begin{array}{cccc}
0 & \sqrt{p} & 0 & 0 \\
0 & 0 & 0 & 0 \\
0 & 0 & 0 & \sqrt{p} \\
0 & 0 & 0 & 0
\end{array}\right]
$$


The following notation will be used henceforth to represent the Bell states

$$
\left|\Phi^{ \pm}\right\rangle\left\langle\Phi^{ \pm}\left|\equiv \rho_{ \pm}^{\|} ;\right| \Psi^{ \pm}\right\rangle\left\langle\Psi^{ \pm}\right| \equiv \rho_{ \pm}^{\perp}
$$

It is clear that such an interaction turns the maximally entangled state, originally shared by Alice and Bob into a mixed entangled state,

$$
\tilde{\rho}_{ \pm}^{\|}=\left[\begin{array}{cccc}
1 & 0 & 0 & \pm \sqrt{1-p} \\
0 & 0 & 0 & 0 \\
0 & 0 & p & 0 \\
\pm \sqrt{1-p} & 0 & 0 & 1-p
\end{array}\right]: \widetilde{\rho}_{ \pm}^{\perp}=\frac{1}{2}\left[\begin{array}{cccc}
p & 0 & 0 & 0 \\
0 & 1-p & \pm \sqrt{1-p} & 0 \\
0 & \pm \sqrt{1-p} & 1 & 0 \\
0 & 0 & 0 & 0
\end{array}\right]
$$

It turns out that the fully entangled fraction $f$ of the four resulting mixed states is the same:

$$
f\left(\widetilde{\rho}_{ \pm}^{\|}\right)=f\left(\widetilde{\rho}_{ \pm}^{\perp}\right)=\frac{1}{4}(1+\sqrt{1-p})^{2}=f(\widetilde{\rho})
$$

where

$$
f\left(\tilde{\rho}_{ \pm}^{\|}\right)=\left\langle\Phi^{ \pm}\left|\tilde{\rho}_{ \pm}^{\|}\right| \Phi^{ \pm}\right\rangle ; \quad f\left(\widetilde{\rho}_{ \pm}^{\perp}\right)=\left\langle\Psi^{ \pm}\left|\widetilde{\rho}_{ \pm}^{\perp}\right| \Psi^{ \pm}\right\rangle
$$

Here we note that fully entangled fraction is same as that of entanglement fidelity [17]. Of course this is not always the case, as we will show later when we subject the second qubit to dissipation for possible improvement of the noisy states. Since entanglement fidelity measures how well the entanglement has been preserved while interacting with a noisy channel we conclude that till this end all the four Bell states are equally corrupted. This is also expected as the noisy channel acts locally on one of the qubits whose state given by the reduced density matrix is the same for all the four Bell states.

We now consider the usefulness of the mixed states given by Eq. (15) to perform quantum teleportation. These family of mixed states are useful for teleportation when 
$f(\widetilde{\rho})>1 / 2$ [14, 15], from which one easily obtains the restriction on the parameter $p$

$$
f(\widetilde{\rho})>\frac{1}{2} \quad \forall p<2 \sqrt{2}-2
$$

Eq. (16) simply states that for all values of $p \geq 2 \sqrt{2}-2, f(\widetilde{\rho}) \leq 1 / 2$ the family of mixed

states $\widetilde{\rho}_{ \pm}^{\|}, \widetilde{\rho}_{ \pm}^{\perp}$ so obtained are not useful for teleportation. Here "not useful" is understood as not better than what can be done classically.

\section{Improving the Noisy Bell States by letting the un- affected Qubit to interact with the Local Environ- ment via Amplitude Damping Channel}

In this section we discuss the possible improvement of the noisy states given by Eq. (15) by subjecting the second qubit to similar dissipative interaction.

\subsection{Damping Parameters same for the two qubits}

We now allow Alice's qubit to interact with the environment via the amplitude damping channel. Here we also assume that the strength of dissipation affecting qubits locally is the same for both. This is not a necessary assumption but only simplifies the degree of algebraic complexity though it does not capture all the intricacies involved. The general case where the damping parameter is different for the two qubits will be treated in the next subsection.

This interaction is described by the following transformation:

$$
\widetilde{\rho}(p) \longrightarrow \widetilde{\widetilde{\rho}}(p)=S^{\prime}(\widetilde{\rho})=W_{0}^{\prime} \rho W_{0}^{\prime \dagger}+W_{1}^{\prime} \rho W_{1}^{\prime \dagger}
$$


where $W_{i}^{\prime}=K_{i} \otimes I$ and $\widetilde{\rho}$ is the density operator representing one of the noisy Bell states: $\left\{\widetilde{\rho}_{ \pm}^{\|}, \widetilde{\rho}_{ \pm}^{\perp}\right\}$

The fully entangled fraction of the $\widetilde{\widetilde{\rho}}$ states are now given by:

$$
\begin{aligned}
& f\left(\widetilde{\widetilde{\rho}}_{ \pm}^{\|}\right)=1-p+\frac{1}{2} p^{2} \\
& f\left(\widetilde{\widetilde{\rho}}_{ \pm}^{\perp}\right)=\begin{array}{c}
1-p \quad \forall p \leq \frac{2}{3} \\
\frac{p}{2} \quad \forall p \geq \frac{2}{3}
\end{array}
\end{aligned}
$$

First we check if there is a range of $p$ such that $f(\widetilde{\widetilde{\rho}})>1 / 2$. If so then we would like to know if that range has an overlap with the range $p \geq 2 \sqrt{2}-2$ because only then that would imply that further application of a dissipative interaction can indeed enhance the teleportation fidelity of the "one qubit affected" noisy states that are formed via the interaction defined by Eq. (10). It is easy to check that

$$
\begin{array}{ccc}
f\left(\widetilde{\widetilde{\rho}}_{ \pm}^{\|}\right) & >\frac{1}{2} & \forall p, p \neq 1 \\
f\left(\widetilde{\widetilde{\rho}}_{ \pm}^{\perp}\right)= & \forall p<\frac{1}{2} \\
\frac{p}{2} & <\frac{1}{2} & \forall p, p \neq 1
\end{array}
$$

Recall that for all $p \geq 2 \sqrt{2}-2, f(\widetilde{\rho}) \leq 1 / 2$. Since from Eq. (20) it follows that $f\left(\widetilde{\widetilde{\rho}}_{ \pm}^{\|}\right)>$ $\frac{1}{2} \forall p, p \neq 1$, therefore $f\left(\widetilde{\widetilde{\rho}}_{ \pm}^{\|}\right)>\frac{1}{2} \quad \forall p \geq 2 \sqrt{2}-2$ which implies that teleportation fidelity of all non teleporting states $\widetilde{\rho}_{ \pm}^{\|}(p) ; p \geq 2 \sqrt{2}-2$ can indeed be enhanced by subjecting the second qubit to dissipation. Here we also note that $f\left(\widetilde{\widetilde{\rho}}_{ \pm}^{\|}\right) \geq f\left(\widetilde{\rho}_{ \pm}^{\|}\right) \forall p \geq 0.80585$. This implies that teleportation fidelity of the teleporting states $\widetilde{\rho}_{ \pm}^{\|}(p) ; 0.80585 \leq p<2 \sqrt{2}-2$ can also be enhanced by this method. Thus the two-qubit affected state $\widetilde{\widetilde{\rho}}_{ \pm}$is better (i.e. improved) with a higher teleportation fidelity than single qubit affected state $\widetilde{\rho}_{ \pm}^{\|}$whenever $p>0.80585$. This is surprising in the sense that the dissipative interaction which spoils 
entanglement in the first step is utilised to improve the quality of the mixed state by applying it to the second subsystem.

However Eqs. (19) and (21) show that the same prescription though curious doesn't

work to turn non teleporting states into teleporting ones. Although $f\left(\widetilde{\widetilde{\rho}}_{ \pm}^{\perp}\right)=p / 2 \geq$ $f\left(\widetilde{\rho}_{ \pm}^{\perp}\right) \forall p \geq 8 / 9$, the enhancement is not sufficient to transform non teleporting states to teleporting states as $f\left(\widetilde{\widetilde{\rho}}_{ \pm}^{\perp}\right)=p / 2$ is always less than or equal to $\frac{1}{2}$.

It may be worth stressing that in showing the above effect we have taken the damping parameter to be the same for both the qubits. We will now consider the case where the damping parameters are different.

\subsection{Damping parameters different for the two qubits}

Let us now treat the problem in a more general way. Here, as before, we will assume that first Bob's qubit undergoes dissipative interaction with the environment and the parameter $p$ of the amplitude damping channel will now be denoted by $p_{b}$. After that we allow the qubit that belongs to Alice also to interact with the environment via amplitude damping channel and the channel parameter now taken to be different from $p_{b}$, is denoted by $p_{a}$. Nevertheless the conclusion remains the same except for the fact that in this case we have the freedom of varying the strength of dissipation for the second qubit which allows us to maximize the enhancement of teleportation fidelity.

The fully entangled fraction $f(\widetilde{\rho})$ of the resulting mixed state $\widetilde{\rho}\left(p_{b}\right)$ formed via interaction given by Eq. (10) is,

$$
f\left(\widetilde{\rho}_{ \pm}^{\|}\right)=f\left(\widetilde{\rho}_{ \pm}^{\perp}\right)=\frac{1}{4}\left(1+\sqrt{1-p_{b}}\right)^{2}=f(\widetilde{\rho})
$$

This is same as Eq. (14), the only difference is that $p$ is now denoted by $p_{b}$. Now when we allow Alice's qubit also to interact with the environment described by Eq. $(17), \widetilde{\rho}\left(p_{b}\right)$ 
states are transformed to $\widetilde{\widetilde{\rho}}\left(p_{a}, p_{b}\right)$.

Irrespective of the source state the fully entangled fraction is the same when only one qubit is affected. The remaining analysis involving subjection of the second qubit to dissipation for possible enhancement of teleportation fidelity is divided into two parts.

The first one corresponds to the cases when the source states are $\left|\Phi^{ \pm}\right\rangle\left\langle\Phi^{ \pm}\right| \equiv \rho_{ \pm}^{\|}$and the second part corresponds to the cases when the source states are $\left|\Psi^{ \pm}\right\rangle\left\langle\Psi^{ \pm}\right| \equiv \rho_{ \pm}^{\perp}$.

\subsubsection{Part 1}

Let us first recall that for $p_{b} \geq 2 \sqrt{2}-2, f(\widetilde{\rho}) \leq 1 / 2$, i.e., the states $\widetilde{\rho}_{ \pm}^{\|}$are non teleporting. After the second interaction for which the damping parameter is $p_{a}$, the fully entangled fraction corresponding to the $\widetilde{\widetilde{\rho}}_{ \pm}^{\|}\left(p_{a}, p_{b}\right)$ states are given by:

$$
f\left(\widetilde{\widetilde{\rho}}_{ \pm}^{\|}\right)=\frac{1}{4}\left[p_{a} p_{b}+\left(1+\sqrt{\left(1-p_{a}\right)\left(1-p_{b}\right)}\right)^{2}\right]
$$

\subsubsection{Condition for "two qubit affected" state having larger teleportation fidelity than the "one qubit affected" state}

Our objective is to obtain the condition such that the inequality

$$
f\left(\rho^{\prime \prime}\right)>f\left(\rho^{\prime}\right)
$$

is satisfied. To be precise we are now looking for those values of $p_{a}$, such that for $p_{b} \geq$ $2 \sqrt{2}-2$, the previous inequality is satisfied. It turns out that for

$$
p_{a}<\frac{4\left[\sqrt{1-p_{b}}\left(2 p_{b}-1\right)-\left(1-p_{b}\right)\right]}{\left(2 p_{b}-1\right)^{2}}=g\left(p_{b}\right)
$$

the inequality $f\left(\rho^{\prime \prime}\right)>f\left(\rho^{\prime}\right)$ is satisfied. However it remains to be ensured that a range of values of $p_{a}$ is obtained for $p_{b} \geq 2 \sqrt{2}-2$. First observe that $g(3 / 4)=0$. This in 
turn implies we can always find some suitable range of $p_{a}$ such that the inequality (20) is satisfied provided $p_{b}>3 / 4$. Stated more explicitly this means that only when Bob's qubit interacted with the environment via the amplitude damping channel characterized by the parameter $p_{b}>3 / 4$, then the possibility of improving the corrupted state by allowing the other qubit to interact, arises. Hence the two qubit affected states are thereby made better than the one qubit affected state. One may also note that not only non teleporting states can be made teleporting but also teleporting states with poor fidelity are made better. We now look for the condition when maximum enhancement of teleportation fidelity is achieved.

\subsubsection{Maximum achievable teleportation fidelity}

Let us now assume $p_{b}>3 / 4$. Thus we have at our disposal a range of possible values of $p_{a}$ satisfying (24) and the inequality (25). Then another important question remains to be answered: For which value of $p_{a}, f\left(\rho^{\prime \prime}\right)$ is the maximum? That is, for which value $p_{a}$ highest fidelity for teleportation can be achievd?

One can easily check that (23) is maximised for

$$
p_{a}=\frac{p_{b}\left(4 p_{b}-3\right)}{\left(2 p_{b}-1\right)^{2}}
$$

Here it is interesting to note that (26) immediately gives a lower bound on the value of the parameter $p_{b}$, which is $3 / 4$ and this bound we have obtained before. Substituting (26) in (23) one obtains the maximum value of fully entangled fraction that can be achieved:

$$
f_{\text {max }}\left(\rho^{\prime \prime}\right)=\frac{p_{b}^{2}}{2\left(2 p_{b}-1\right)}
$$

One should note that $f_{\max }>1 / 2$.

\subsubsection{Non teleporting states can always be made teleporting}

To begin with Alice and Bob initially shared a maximally entangled state. If Bob's 
qubit gets affected by the $\mathrm{ADC}, p_{b}>3 / 4$ then the fidelity of the resulting noisy state can be improved by subjecting the unaffected qubit to similar dissipation characterized by the parameter $p_{a}$ in accordance with Eq. (25). The maximum "fully entangled fraction" or the maximum fidelity of teleportation is obtained by substituting (26) in the expression (23).

Since for $p_{b} \geq 2 \sqrt{2}-1$ the state $\rho^{\prime}\left(p_{b}\right)$ is not capable of teleportation better than what can be achieved classically. It is now clear that those non teleporting states can always be made teleporting by allowing the other qubit to interact with the environment with an appropriate choice of the parameter $p_{a}$. In particular the maximum fidelity can be obtained if we choose $p_{a}$ in accordance with (26). Even if the state $\rho^{\prime}\left(p_{b}\right)$ is a teleporting one (which it is, if $\left.3 / 4<p_{b}<2 \sqrt{2}-2\right)$ still its fidelity can be enhanced by allowing the other qubit to interact with the environment for any value of $p_{a}$ defined by $(25)$.

\subsubsection{Part 2}

We now analyze what happens when the source states are the superposition of antiparallel spins. First we note that when one qubit is affected the fully entangled fraction is given by Eq. (10) or Eq. (22). After the second qubit is affected, the interaction being governed by an appropriate superoperator, the density operator of the mixed state is given by

$$
\widetilde{\widetilde{\rho}}_{ \pm}^{\perp}=\left[\begin{array}{cccc}
p_{a}+p_{b} & 0 & 0 & 0 \\
0 & 1-p_{b} & \pm \sqrt{\left(1-p_{a}\right)\left(1-p_{b}\right)} & 0 \\
0 & \pm \sqrt{\left(1-p_{a}\right)\left(1-p_{b}\right)} & 1-p_{a} & 0 \\
0 & 0 & 0 & 0
\end{array}\right]
$$

Now recall the definition of fully entangled fraction. One can easily see that two possible candidates for fully entangled fraction are 


$$
\begin{aligned}
& f_{1}=\frac{p_{a}+p_{b}}{4} \\
& f_{2}=\frac{1}{4}\left(\sqrt{1-p_{a}}+\sqrt{1-p_{b}}\right)^{2}
\end{aligned}
$$

The fully entangled fraction is given by $\max \left[f_{1}, f_{2}\right]$. Whether $f_{1}>f_{2}$ or vice versa depends on the particular values of $p_{a}$ and $p_{b}$, i.e., whether $f_{1}$ is the fully entangled fraction or $f_{2}$ is depends on the specific choices of the damping parameters.

Let us first assume that $f_{1}>f_{2}$. Now observe that $f_{1} \leq 1 / 2$. Therefore teleportation fidelity can never exceed $2 / 3$. Hence non teleporting states can never become teleporting.

Now if $f_{2}>f_{1}$, then note that $f_{2}\left(p_{a}, p_{b}\right) \leq f\left(\widetilde{\rho}_{ \pm}^{\perp}\right) \forall p_{a}, p_{b}$. Therefore whatever be the case the above two observations guarantee that by subjecting the second qubit to a similar dissipative interaction, the non teleporting states cannot be made teleporting.

\section{Remarks and Conclusion}

We know that given any mixed state the corresponding teleportation fidelity has contribution from two parts: (a) the quantum part i.e. the shared entanglement and (b) the classical part, i.e. the classical correlations. Since entanglement cannot be increased by TPLOCC, an enhancement in teleportation fidelity suggests that, for the states on the border line of non teleporting and teleporting, it is the classical part that contributes more heavily than the quantum part so that even a non teleporting state becomes teleporting in spite of losing entanglement. Thus TPLOCC which spoils entanglement but nevertheless can sometimes redistribute the classical correlations in a helpful way.

We now discuss the implications of our result in the context of distilling entanglement from the noisy states. For distillation [9] of mixed entangled states having fully entangled 
fraction $f \leq \frac{1}{2}$ one needs to apply filtering methods [12, 13] so that $f$ exceeds $\frac{1}{2}$. This is important because only then the distillation protocol of Bennett et al [9] could be applied. However filtering being non trace preserving involves measurement followed by post selection which necessarily discards a large number of available pairs. In view of this our results provide a significant improvement for a large class of states for which fully entangled fraction can be made greater than half without resorting to filtering implying that we need not discard pairs.

We have shown that the noisy entangled states constructed from the source states by similar (meaning same for all the source states) dissipative interactions, can indeed have very different properties even though the source states are local unitary equivalent. This observation might be helpful while trying to establish entanglement when the noisy quantum channel is the amplitude damping channel. If we are provided with either of the states $\left|\Psi^{ \pm}\right\rangle$then instead of trying to share it we may first convert it to $\left|\Phi^{ \pm}\right\rangle$state by applying pauli rotations locally. The reason is even if the entanglement gets spoiled at its worse so that the mixed states are non teleporting we can still make them suitable for teleportation by TPLOCC. Besides being made useful for teleportation these states also allow for direct application of the Bennett protocol for distillation [9]. Thus our results do indicate that a priori knowledge of the noisy channel may decide sometimes which Bell state is to be shared. One of course should try to share the case specific Bell state for which the resulting mixed states allow for further useful manipulations.

I would like to thank Daniel Terno, Ujjwal Sen and Guruprasad Kar for helpful discussions.

\section{References}


[1] P. Badziag, M. Horodecki, P. Horodecki and R. Horodecki, LANL preprint, quant-ph/ 9912098.

[2] E. Schrodinger, Naturwissenschaften 23, 807 (1935); 23, 823 (1935); 23, 844 (1935);

For a review see: M. B. Plenio and V. Vedral, Cont. Phys. 39, 431 (1998); Lecture notes of Lucien hardy available at http://www.qubit.org.

[3] A. Einstein, B. Podolski, and N. Rosen, Phys. Rev. 47, 777 (1935) [reprinted in Quantum theory and Mesaurement, edited by J. A. Wheeler and W. Z. Zurek (Princeton University Press, Princeton, 1983)].

[4] An up to date discussion on quantum nonlocality can be found in D. Home, Conceptual Foundations of Quantum Mechanics: An overview from modern perspective (Plenum Press, New York), Ch. 4.

[5] J. S. Bell, Physics 1, 195 (1964) [reprinted in J. S. Bell, Speakable and Unspeakable in Quantum Mechanics (Cambridge University Press, Cambridge, 1987), p. 14].

[6] C. H. Bennett, Physics Today 48, 24 (1995).

[7] J. Preskill, Proc. Roy. Soc. A: Math., Phys. and Eng. 454, 469 (1998); A brief but excellent article is by R. Jozsa, quant-ph/9707034.

[8] C. H. Bennett, G. Brassard, C. Crepeau, R. Jozsa, A. Peres and W. K. Wootters, Phys. Rev. Lett. 70, 1895 (1993).

[9] C. H. Bennett, G. Brassard, S. Popescu, B. Schumacher, J. A. Smolin and W. K. Wootters, Phys. Rev. Lett. 76, 722 (1996); D. Deutsch, A. Ekert, R. Jozsa, C. Macchiavello, S. Popescu and A. Sanpera, Phys. Rev. Lett. 77, 2818 (1996)

[10] C. H. Bennett, C. A. Fuchs and J. A. Smolin, quant-ph/9611006. 
[11] C. H. Bennett, D. P. DiVincenzo, J. A. Smolin and W. K. Wootters, Phys. Rev. A 54, 3814 (1997).

[12] N. Gisin, Phys. Lett. A, 210, 151 (1996).

[13] C. H. Bennett, H. J. bernstein, S. Popescu and B. Schumacher, Phys. Rev. A, 53, 2046 (1996).

[14] R. Horodecki, M. Horodecki and P. Horodecki, Phys. Lett. A, 222, 21 (1996).

[15] M. Horodecki, P. Horodecki and R. Horodecki, Phys. Rev. A, 60, 1888 (1999).

[16] J. Preskill, http://www. theory. caltech. edu/ people/ preskill/ ph229.

[17] B. Schumacher, Phys. Rev. A 54, 2614 (1996). 